\title{
(2) OPEN ACCESS \\ Successful return to professional men's football (soccer) competition after the COVID-19 shutdown: a cohort study in the German Bundesliga
}

\author{
Tim Meyer (1), ${ }^{1}$ Dietrich Mack, ${ }^{2}$ Katrin Donde, ${ }^{2}$ Oliver Harzer, ${ }_{1}^{2}$ Werner Krutsch, ${ }^{3}$ \\ Annika Rössler, ${ }^{4}$ Janine Kimpel (i) , ${ }^{4}$ Dorothee von Laer (i), ${ }^{4}$ Barbara C Gärtner ${ }^{5}$
}

Institute of Sports and

Preventive Medicine, Saarland University, Saarbrücken,

Saarland, Germany

${ }^{2}$ Bioscientia Laboratory

Ingelheim, Ingelheim, Germany

${ }^{3}$ Department of Trauma

Surgery, Universitatsklinikum

Regensburg, Regensburg,

Germany

${ }^{4}$ Institute of Virology, Department of Hygiene,

Microbiology and Public Health, Medical University Innsbruck,

Innsbruck, Austria

5 Institute of Medical

Microbiology and Hygiene,

Universitatsklinikum des

Saarlandes und Medizinische

Fakultat der Universitat des

Saarlandes, Homburg, Saarland,

Germany

\section{Correspondence to}

Professor Tim Meyer, Institute of Sports and Preventive Medicine, Saarland University

- Campus Saarbrucken, 66123

Saarbruecken, Saarland,

Germany;

tim.meyer@mx.uni-saarland.de

Accepted 26 August 2020

Published Online First

24 September 2020
Check for updates

(c) Author(s) (or their employer(s)) 2021. Re-use permitted under CC BY-NC. No commercial re-use. See rights and permissions. Published by BMJ.

To cite: Meyer T,

Mack D, Donde K,

et al. Br J Sports Med

2021:55:62-66.

\section{ABSTRACT}

Objectives To evaluate the restart of the German Bundesliga (football (soccer)) during the COVID-19 pandemic from a medical perspective.

Methods Participants were male professional football players from the two highest German leagues and the officials working closely with them. Our report covers nine match days spread over 9 weeks (May to July 2020). Daily symptom monitoring, PCR testing for SARS-CoV-2 RNA twice weekly, and antibody tests (on two occasions - early during the phase in May 2020 and in the week of the last match) were conducted. Target variables were: (1) onset of typical COVID-19 symptoms, (2) positive PCR results, and (3) IgG seroconversion against SARS-CoV-2. All detected seroconversions were controlled by neutralisation tests. Findings Suspicious symptoms were reported for one player; an immediate additional PCR test as well as all subsequent diagnostic and antibody tests proved negative for coronavirus. Of 1702 regularly tested individuals (1079 players, 623 officials members), 8 players and 4 officials tested positive during one of the first rounds of PCR testing prior to the onset of team training, 2 players during the third round. No further positive results occurred during the remainder of the season. 694 players and 291 officials provided two serum samples for antibody testing. Nine players converted from negative/borderline to positive (without symptoms); two players who initially tested positive tested negative at the end of the season. 22 players remained seropositive throughout the season. None of the seroconversions was confirmed in the neutralisation test. Conclusion Professional football training and matches can be carried out safely during the COVID-19 pandemic. This requires strict hygiene measures including regular PCR testing.

\section{INTRODUCTION}

There has been considerable debate about the desirability of a restart of professional sport during the COVID-19 pandemic, in particular football (soccer). ${ }^{12}$ Of course, players and clubs have an interest in continuing their professional activities. However, proper safety precautions have to be put in place to ensure the health of players and officials (and requirements for proper infection protection for all citizens) and the integrity of competition. ${ }^{3}$

Due to the COVID-19 pandemic the 2019/20 season of the German first professional football league ('Bundesliga') was interrupted after the match between Mönchengladbach and Cologne on 11 March 2020. On 17 March the DFB's (German national football association) medical committee made recommendations for home-based training and management of players with suspected COVID-19 disease as well as for training in small groups. Two weeks later the DFL formally appointed the Sports Medicine/Special Match Operations Task Force: four medical doctors, including co-authors of this paper (TM, WK and BG), and two employees each of the DFL (Deutsche Fußball Liga/ German football league) and the DFB.

Tracing of past COVID-19 cases and their circumstances in the highest three German men's leagues and in the women's first league was immediately started. During the next weeks, the Bundesliga Hygiene Protocol (BHP) was developed and, after slight amendments (https://www.dfl.de/en/topics/fixtureplanning/sports-medicine-special-match-operationstask-force-concept), this was approved by responsible federal institutions (Ministry of Health, employers mutual insurance association) in late April. Regular team training recommenced in early May and the two highest German men's leagues (Bundesliga and Bundesliga 2) restarted on 16 May; 2 weeks later the women's Bundesliga began as did the third men's league.

The BHP was based on tracing all recorded COVID-19 cases, as well as two additional principles. The protocol relied on:

1. implementation of strict hygiene (eg, masks, disinfection) and physical distance rules for training, matches (eg, symptom questionnaire and temperature measurements before entry into the stadium/ training site), travelling, hotel accommodation and private life, including a 7 day team quarantine before the continuation of the season;

2. repeated reverse transcription polymerase chain reaction (RT-PCR) testing of pharyngeal swabs in all players and club officials with close contact to players.

This study evaluates the success of the concept based on available objective indicators, that is, symptoms, testing results and development of antibodies against SARS-CoV-2 (seroconversion). These findings may help clinical colleagues in other sports and other parts of the world as they address the question of return to play during and after the pandemic.

\section{METHODS}

Study design and ethics

This was a 2 month prospective observational study in 36 professional men's football teams (all teams of the Bundesliga and Bundesliga 2; third league and women's league were managed by the task force, too, but did not participate in the study) in 


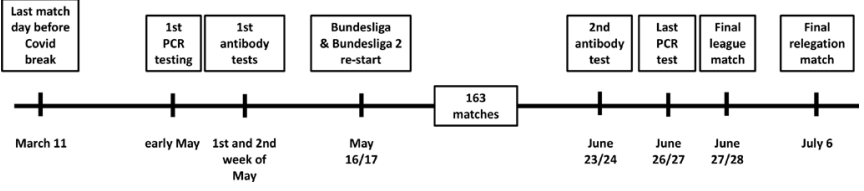

Figure 1 Time schedule from the interruption of the German professional football season 2019/20 until its successful termination. $P C R$, polymerase chain reaction.

Germany to assess the safety of a battery of measures to avoid SARS-CoV-2 infections and transmissions. Ethical approval was obtained from the local ethics committee (Registration 202010523_2 Landeskammer Rheinland-Pfalz, Germany). The timeline is illustrated in figure 1 .

In each of the two highest German men's leagues, 18 teams compete over 34 match days after which the final result appears in the form of a table. The season was interrupted due to the COVID-19 pandemic after the 25th match day in both leagues. Players provided written informed consent to participate in antibody testing (see below) and to give blood for that purpose. It was explained to them that antibody testing only served as a scientific accompaniment of the season. All other routine measures were considered part of the medical care as agreed with the clubs in each player's individual contract.

\section{Symptom monitoring}

Team physicians monitored players' symptoms using a questionnaire provided in a mobile app. Players were also encouraged to make direct phone contact with their team doctors as needed. The questions covered all typical COVID-19 symptoms (cough, fever, smell and taste sensations, difficulties breathing, etc) as well as general ones for respiratory infections, for example, sore throat, running nose, malaise. Team doctors were free to use their self-designed questionnaires or applications.

It is noteworthy that other groups of individuals were affected by the same or analogous measures: referees (included in PCR testing), ball boys, doping control officers, TV personnel. A hygiene officer (medical doctor) responsible for the implementation of the concept had to be named by each club. All clubs were asked before the onset of regular team training to identify individuals around the team with risk factors for severe courses of COVID-19 (eg, age $>65$ years, pre-existent diseases of the respiratory or cardiovascular systems). It was recommended to exclude them from participation in training and match activities or at least-in case of indispensability - to thoroughly inform them about medical risks. The same policy was used for the players regardless of known diseases.

\section{PCR testing}

All players provided pharyngeal swabs (oropharyngeal, nasopharyngeal, or both) for PCR testing for SARS-CoV-2 RNA twice within 2-5 days before the onset of regular team training: two negative tests served as "entry ticket" for regular team training. Players were swabbed twice per week thereafter. During the entire season, the last samples before each match were taken on the day before the match. Swabs were taken by trained medical personnel. All samples were analysed in certified medical laboratories with carefully monitored quality control. Results were returned within 24 hours, that is, on the morning of the match day at the latest. However, due to the large area to be covered and the short turnaround times, different laboratories were involved using one of the following assays:
- SARS-CoV-2 Test CE-IVD, Roche, Basel, Switzerland (target genes: ORF1a/1b, E)

- RealStar SARS-CoV-2 RT-PCR Kit 1.0, Altona, Hamburg, Germany (target genes: S, E)

- Ridagene SARS-CoV-2-PCR, R-Biopharm, Darmstadt, Germany (target gene: E).

\section{Antibody testing}

Venous blood samples for antibody testing were taken from an antecubital vein in the supine or sitting position after appropriate rest. Participants provided samples twice during the entire season: once together with the third or fourth PCR testing (in the first and second week of May 2020) and once together with the second-last PCR testing during the season (23 or 24 June 2020).

SARS-CoV-2 specific immunoglobulin G (IgG) antibodies were determined to identify seroconversion as a measure for contact with SARS-CoV-2. Players were not excluded from further PCR testing once they had a positive antibody result. All antibody determinations were carried out in a single laboratory (Bioscientia Laboratory Ingelheim, Ingelheim, Germany).

Anti-SARS-CoV-2 IgG antibodies in human sera were detected by EUROImmun anti-SARS-CoV-2-ELISA (IgG) (EUROimmun, Lübeck, Germany) using recombinant spike protein domain S1 including the immunologically relevant receptor-binding domain RGD. ELISA tests were processed using the fully automated EUROimmune EUROLabWorkstation ELISA (EUROimmun) according to the protocol given by the manufacturer. Each ELISA run also contained positive and negative control sera and the calibrator. Values given were the ratio of optical density (OD) readings of the sample or controls and the calibrator. Ratios $<0.8$ were considered negative, ratios between 0.8 and 1.1 were borderline, and ratios $>1.1$ were positive. According to the manufacturer, the assay had a specificity of $>99 \%$. The sensitivity varied according to the study populations tested, but reached $>75 \%$ for patients 10-20 days after infection and 93.8\% for sera $>20$ days after infection (EUROimmun testing brochure).

\section{Neutralisation tests}

All sample pairs indicating a seroconversion, that is, turning from negative into positive or from borderline into positive, were investigated by a neutralisation test to finally clarify whether a SARS-CoV-2 contact had led to this change. A neutralisation assay based on a replication defective vesicular stomatitis vector (VSV) pseudotyped with the spike protein of SARS-CoV-2 was used. VSV* $\Delta G$ encoding green fluorescent protein (GFP) as marker gene was produced on $293 \mathrm{~T}$ cells expressing a SARS-CoV-2 spike. For the neutralisation assay, serum samples were heat inactivated for $30 \mathrm{~min}$ at $56^{\circ} \mathrm{C}$ and subsequently diluted fourfold in complete medium in duplicates. VSV $* \Delta$ G-S particles, corresponding to 100-200 infected cells per 96-well in non-neutralised control wells, were added and serum/virus mixes were incubated for 1 hour at $37^{\circ} \mathrm{C}$. Subsequently, $\sim 70 \%$ confluent $293 \mathrm{~T}$ cells, overexpressing human ACE-2, were infected with the mixes. The following day, GFP positive cells were counted using an ImmunoSpot S6 Ultra-V reader and FluoroSpot software (CTL Europe GmbH, Bonn, Germany). Fifty percent neutralisation titres were defined as the last serum dilution where the mean spot number of duplicate samples was smaller than half of the mean spot number in virus only wells (quadruplicates). Titres $\leq 1: 4$ were considered negative, and titres of 1:16 or above were considered positive. 


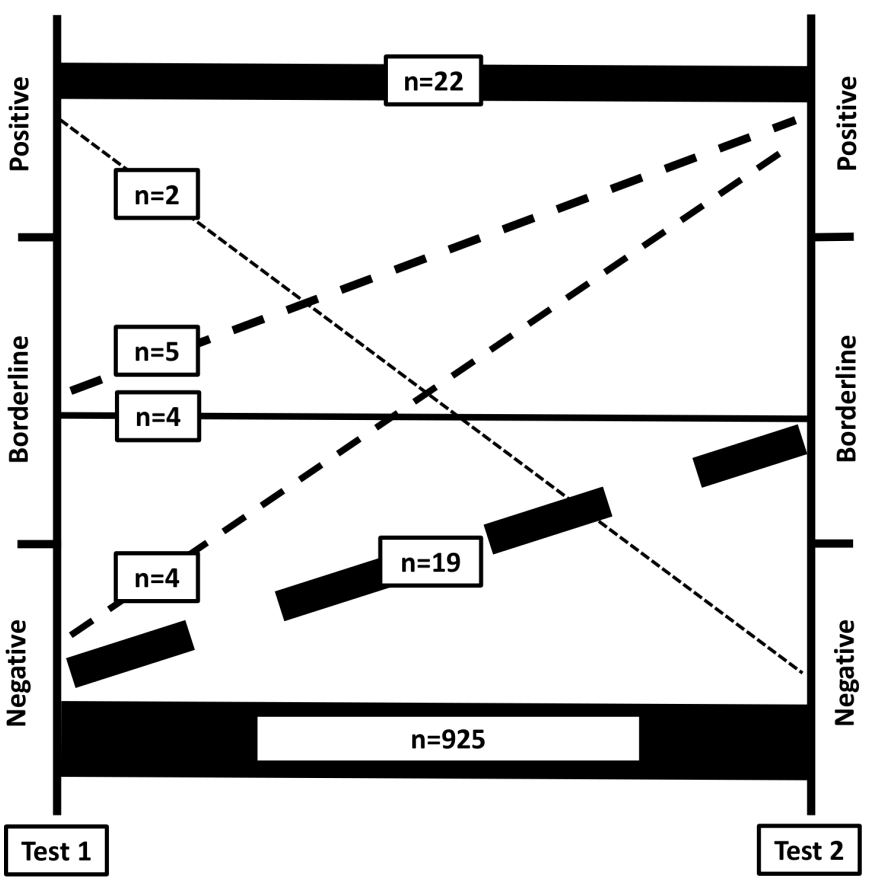

Figure 2 Schematic depiction of antibody developments between restart of team training and termination of the season. Width of bands is proportional to the number of cases (except for constantly negative tests for which the band is reduced by a factor 20); concrete numbers are given explicitly for each band. Solid bands indicate unchanged situations, interrupted bands indicate changes.

\section{Statistics}

We report descriptive statistics. Raw numbers are provided for different outcomes of the tests. For some dichotomous variables, percentages have been calculated.

\section{RESULTS}

The German football season finished in both leagues within the adapted time frame, that is, the last match days were on 27 June (Bundesliga) and 28 June (Bundesliga 2), with the relegation matches taking place on 2 and 6 July 2020. Two matches of one club had to be postponed due to positive PCR results and consecutive team quarantine before restart of the league (see below).

\section{Symptoms}

No suspicious symptoms were reported by the team physicians for any player except for one (rhinitis, sore throat) in early June who was immediately tested for SARS-CoV-2 RNA. The test revealed a negative result as well as all later PCR tests and the final antibody screening.

\section{PCR testing}

Altogether, 1702 individuals were regularly tested (898 from the Bundesliga and 804 from the Bundesliga 2). This number included 1079 players (550 and 529) and 623 officials (348 and 275). Eight players and four officials from a total of seven clubs were tested positive during one of the first two rounds (before the onset of team training) and put into quarantine, that is, they were isolated from all other team members. Three of all positive cases were later proven to be remnants of earlier COVID-19 infections based on medical documents provided by the team doctors (eg, symptom history, earlier PCR results, antibody results). There was no obvious regional pattern among
Table 1 Optical density (OD) values for all antibody pairs compatible with "seroconversion"

\begin{tabular}{lllll}
\hline $\begin{array}{l}\text { Seroconversion } \\
\text { (negative to positive) }\end{array}$ & & $\begin{array}{l}\text { Seroconversion } \\
\text { (borderline to positive) }\end{array}$ \\
\cline { 1 - 1 } $\begin{array}{l}\text { OD first test } \\
\text { result }\end{array}$ & $\begin{array}{l}\text { OD second test } \\
\text { result }\end{array}$ & & $\begin{array}{l}\text { OD first test } \\
\text { result }\end{array}$ & OD second test result \\
\hline 0.13 & 1.33 & 1.04 & 1.15 \\
0.55 & 1.62 & 0.91 & 2.20 \\
0.61 & 1.51 & 0.86 & 1.61 \\
0.30 & 1.89 & 0.96 & 1.51 \\
\hline
\end{tabular}

the positive results. After returning to team training, two more positive results (both players) occurred during the third round of testing: one team was sent into quarantine for 2 weeks and frequently retested by health authorities, and one of these cases was shown to result from a proven earlier COVID-19 infection. No further positive test results occurred during the remaining season.

\section{Antibody testing}

Finally, 2169 serum samples were examined of which 1970 (1388 from 694 players; 582 from 291 officials) were paired ones from pre- and post-testing (and, thus, subjects for this study), that is, 199 players or officials only provided one sample. Three clubs (two from the Bundesliga and one from the Bundesliga 2) did not participate in the antibody part of the study for reasons unrelated to the study targets (difficult organisation, earlier internal antibody testing, etc). Although it is likely that borderline results have to be considered as negative in most cases, they are listed separately.

During the initial testing period, there were nine borderline and 24 positive test results from 19 clubs, among which were 30 players. During re-testing, we found 23 borderline and 31 positive results from 21 clubs (45 players). Figure 2 illustrates these findings in more detail. The highest number of non-negative (borderline or positive) tests in a single club was three (all of them positively tested players) during the first testing and six during re-testing (all of them players, three of them borderline). Altogether, 33 individuals (3.4\%) tested positive during either the initial testing $(n=24 ; 2.4 \%)$ or re-testing $(n=31 ; 3.2 \%)$.

The OD ratios of the seroconversions, that is, ones which changed from negative or borderline into positive antibody results, are listed in table 1 . None of these nine seroconversions (four from negative, five from borderline to positive) was confirmed by neutralisation tests, that is, all 18 neutralisation tests were negative.

\section{DISCUSSION}

We demonstrated that training and playing football with strict hygiene precautions was not associated with infections with SARS-CoV-2 under the epidemiological circumstances that were present in Germany during the months of May and June 2020. One hundred and sixty-five matches were played and this resulted in no player or official: (1) suffering a documented infection during the season; (2) being infected with SARS-CoV-2 (as shown by RT-PCR) throughout the season; or (3) seroconverting (IgG antibodies for COVID-19). These results are meaningful as they indicate that professional outdoor football is feasible during the ongoing COVID-19 pandemic.

How should we assess the success or failure of the Bundesliga hygiene protocol? Practitioners may regard the fact 
that teams were able to complete the season as a very meaningful criterion. From a more scientific perspective, symptom monitoring can only be used as a rough indicator of SARS-CoV-2 transmission. This approach is particularly weak in a young population like this one with frequent mild courses of the disease. ${ }^{4}$ Therefore, repeated PCR testing was initiated which represents the current "gold standard" to assess contagiousness ${ }^{5}$ and its frequency has been deemed crucial based on simulations. ${ }^{6}$ However, even with a testing density as high as in this study, it is possible that a few infected players might be overlooked if they "bypassed" the testing time point or were missed by sample collection (or both). This led to the addition of IgG antibody testing which can be considered the most comprehensive approach to detect infectious episodes in the past (but not acute ones, of course).

\section{Serology results}

The fact that over 99\% of the participating players and officials maintained their serology status (positive or borderline/negative) is already reassuring that no extensive SARS-CoV-2 transmission occurred. However, it is of particular interest to have a closer look at those individuals who changed their status in an "upward manner", that is, from negative/borderline to positive. This occurred in nine individuals, among them four from negative to positive. Besides spontaneous fluctuations of the antibody concentrations, ${ }^{7}$ an infection during the weeks before the initial test would be a likely explanation. This would mean that antibody concentrations were still on the rise at the initial testing and reached positive levels at the end of the season. An undetected infection during the season may be regarded as an alternative explanation. However, this must have escaped all PCR tests and not have led to any infection of teammates or opponents as indicated by their negative results throughout.

Finally, and most importantly, neutralisation tests which can be regarded as the gold standard for assessing immunity have not confirmed any seroconversion. Therefore, it seems most likely that the cut-off value of the utilised antibody test was set very "sensitive" and may have impaired specificity. The relatively small differences in the OD ratio between test time points for all nine sample pairs speaks in favour of this.

At first sight, a change from positivity to negativity (as happened in two players) does not make sense, but a loss in antibody concentrations over longer periods of time is a well-known phenomenon in viral immunology, for example, for hepatitis B and influenza. ${ }^{8}$ Moreover, it could be shown that individuals after mild COVID-19 infections had a reduced level of IgG (and in some patients negative results) compared with patients with severe disease. ${ }^{10}$

\section{Influence from pandemic activity}

Clearly, one cannot extrapolate from our report that our measures would be equally effective in other settings, for example, with other community rates of infection. Our data relate to Germany during the period between May and June 2020. In Germany, the average daily number of newly diagnosed COVID-19 infections was 588 (minimum 192, maximum 1639), ${ }^{11}$ which is equal to about five infections per 100000 inhabitants per week. Under the assumption that a significant proportion of COVID-19 infections remains undiagnosed with a $90 \%$ dark figure, ${ }^{12} 13$ this leads to an estimation of about 50 infections per 100000 inhabitants per week. It needs to be taken into account that our hygiene measures were initiated in March and April when the rate of new infections was much higher and the public fear of getting infected much stronger than later on. It is therefore possible that less strict measures would have elicited the same protective effect. The secular trend for decreasing pandemic activity likely contributed to the successful finalisation of the Bundesliga season without overt SARS-CoV-2 infections.

\section{Football-related transmission?}

No valid statement can be made from our data about the risk of SARS-CoV-2 transmission during football training and match play. The measures used here together aimed to minimise the risk of any contagious player or official being present on the pitch (and in the dressing room-a high-risk environment). All potentially contagious persons - as identified by PCR testing — had been identified during the very first testing rounds and isolated from the other team members to avoid any entry of SARS-CoV-2. Although this was fortunate for the health of the players, it similarly eliminated any chance to assess the likelihood of football-related disease transmission. There are indications that disease transmission during training and match play itself is not very high, ${ }^{14}$ but they come from indirect methods (tracking data with distance measurements) and can neither be confirmed nor falsified here.

\section{"False positives": interpretation difficulties from "past" COVID-19 infections}

The greatest medical challenge for the continuation of the Bundesliga and Bundesliga 2 arose when there were players with positive PCR test results just before the onset of team training. Although it had been communicated before that such an outcome of initial testing was likely, this led to considerable public criticism and challenges of the entire project. In such a large cohort of individuals living in an ongoing COVID-19 pandemic over several weeks, detection of some acute and even more "past" infections is very likely.

Until we began working with the Bundesliga, most of the PCR testing in Germany had been conducted on symptomatic individuals only, that is, the pre-test probability for a positive outcome was much higher than in our population. Therefore, the experience on the laboratory side as well as in the public domain was minimal for a differing testing scenario with a lower pre-test likelihood. A positive PCR result may stem from a prior coronavirus infection, that is, the detected RNA merely reflects remnants on the mucosa which are not contagious anymore. ${ }^{15}$ RNA might be detected over several weeks despite infectivity only lasting for 5 to 9 days. Thus, starting a screening programme in asymptomatic individuals during an ongoing pandemic harbours the risk of identifying past infections that are not infectious any longer but show the same PCR results as acute infections.

Taken together, past or acute infections cannot clearly be distinguished by means of PCR only. As a consequence, a science-orientated committee is needed to decide cases where there is doubt. The presence of antibodies and information from medical and family history may be helpful in this regard. Our experience suggests that it is wise to start testing early to identify such cases when you want to restart a season under COVID-19 pandemic circumstances. Additionally, it is essential to not only use $\beta$-coronavirus specific target genes such as the $\mathrm{E}$ gene but also to include SARS-CoV-2-specific target genes into PCR testing to minimise the risk of "false positives" (in fact, correctly identified Corona-RNA) from contact with other viruses of the Corona family.

"False negatives": implications for test organisation RT-PCR testing in the laboratory is very sensitive but the preanalytical phase, particularly swab taking, has to be considered 


\section{What are the findings?}

- There are preventive strategies to overcome the COVID-19 threat to professional team sports like football (soccer).

- Frequent PCR testing for the presence of SARS-CoV-2 RNA is feasible and proved effective in avoiding COVID-19 transmission in our setting.

\section{How might it impact on clinical practice in the future?}

- Other team sports may consider this protocol or parts of it to restart their leagues.

- Future prevention protocols may learn from difficulties and experiences within the Bundesliga protocol.

critical. ${ }^{16}$ During the early phase of the COVID-19 disease, which is most likely the detectable one in symptom-free football players and officials, the SARS-CoV-2 virus can be expected to mainly replicate on the oropharyngeal and nasopharyngeal mucosa of the throat. ${ }^{15}$ This might change during a later phase of the disease with higher viral loads in samples from the lower respiratory tract. ${ }^{15}$ However, all swabs have to be taken with proper technique. Properly performed swabbing is unpleasant for the patient/athlete. Testing personnel may be reluctant to "harm" well known players once they start to complain. In addition, it is not unthinkable that clubs do not have an interest in losing players for very important matches and, thus, may have an interest in swabs to be taken more "loosely".

Only the employment of external testing staff can completely make up for this, although, as in this study, repeated testing partly addresses this limitation. We authors do not have any evidence that there was "cheating" behaviour (aiming to miss a positive diagnosis). In addition, educational material was provided for all clubs, and they employed only medically educated staff for sample collection. We note that a club that considers such an approach (deliberately not detecting a positive case) would put the health of all other players and officials at risk (and, therefore, its own success).

\section{Study limitations}

Obviously, symptom reporting is the weakest indicator for the presence of COVID-19 disease in such a young population. Thus, it is possible that some underreporting of symptoms was caused due to the feeling that frequent PCR testing guarantees safety.

Although the number of participating players and officials was high (64.3\% of the players and $46.7 \%$ of the officials), not all players participated in antibody testing. Therefore, theoretically there is a risk of seroconversions that may have remained undetected. One could even speculate that players who thought that they had COVID-19 disease might have been reluctant to participate. On the other hand, the chance of such players being "antibody-positive" should have been slightly higher, which might have raised their motivation to participate. However, all players and officials had to undergo regular swab testing for SARS-CoV-2 detection by RT-PCR. This definitely makes up for the low degree of uncertainty from limited participation in the antibody part of the study.

IgG was the only type of antibody measured in this study. This could have led to early stages of COVID-19 disease remaining undetected because detecting IgM or IgA is more likely during this period. However, this was not the main purpose for the determination of antibodies here. Finally, based on the measurements presented here, it is impossible to decide which measure was the most effective one to prevent SARS-CoV-2 transmission. This can only be solved by comparisons between two different approaches-a study that may become feasible when more leagues have finished their season.

\section{CONCLUSION}

In one of the leading professional football leagues in the world, competition was successfully reinstated under the circumstances of the COVID-19 pandemic without documented transmission of the infectious disease. This contradicts several warnings and recommendations against playing football. We believe that our application of strict public health measures combined with regular testing were critical.

Acknowledgements We thank all other members of the task force (Markus Braun, Ansgar Schwenken, Andreas Nagel, Jens Busch and Manuel Hartmann) who contributed immensely to the development and instalment of the

"German protocol". Additionally, we are very grateful to the team doctors of all participating teams who supported the study very well. We thank Sarah Kurz and Maximilian Steinacker (Bioscientia Department Clinical Trials) and the entire staff of Bioscientia Labor Ingelheim for the support of this study. Moreover, many thanks to the different laboratories performing the PCR tests all over Germany. We thank Lisa-Maria Raschbichler and Albert Falch for excellent technical support.

Contributors Each author contributed sufficiently to the manuscript to justify authorship. TM, WK and BG were members of the group who designed the hygiene concept. DM, KD and $\mathrm{OH}$ were concerned with laboratory determinations in the Bioscientia Laboratory Ingelheim. DvL, JK and AR are working for the virology lab in Innsbruck which conducted the neutralisation tests. All of us were involved in the conceptualisation of the study and writing and proofreading of the manuscript.

Funding The study was funded by the DFL (Deutsche Fußball Liga).

Competing interests Authors who were members of the task force received a fee for their work in the task force (TM, WK, BG). DM, KD, and OH are employees of Bioscientia Labor Ingelheim, which performed antibody testing and part of the PCRtesting reported in this study.

Patient and public involvement Patients and/or the public were not involved in the design, conduct, reporting or dissemination of the plans of this research.

Patient consent for publication Not required.

Ethics approval Ethical approval was obtained from the local ethics committee (Registration 2020-15023_2 Landesärztekammer Rheinland-Pfalz, Germany).

Provenance and peer review Not commissioned; externally peer reviewed.

Data availability statement No data are available. All original data obtained from the players are fully confidential.

Open access This is an open access article distributed in accordance with the Creative Commons Attribution Non Commercial (CC BY-NC 4.0) license, which permits others to distribute, remix, adapt, build upon this work non-commercially, and license their derivative works on different terms, provided the original work is properly cited, appropriate credit is given, any changes made indicated, and the use is non-commercial. See: http://creativecommons.org/licenses/by-nc/4.0/.

\section{ORCID iDs}

Tim Meyer http://orcid.org/0000-0003-3425-4546

Janine Kimpel http://orcid.org/0000-0002-5210-7905

Dorothee von Laer http://orcid.org/0000-0001-5825-7237

\section{REFERENCES}

1 Corsini A, Bisciotti GN, Eirale C, et al. Football cannot restart soon during the COVID-19 emergency! A critical perspective from the Italian experience and a call for action. Br J Sports Med 2020. doi:10.1136/bjsports-2020-102306. [Epub ahead of print: 24 Mar 2020].

2 Duarte Muñoz M, Meyer T. Infectious diseases and football - lessons not only from COVID-19. Sci Med Football 2020;4:85-6.

3 Carmody S, Murray A, Borodina $M$, et al. When can professional sport recommence safely during the COVID-19 pandemic? Risk assessment and factors to consider. $\mathrm{Br} J$ Sports Med 2020;54:946-8. 


\section{Original research}

4 Baettig SJ, Parini A, Cardona l, et al. Case series of coronavirus (SARS-CoV-2) in a military recruit school: clinical, sanitary and logistical implications. BMJ Mil Health 2020. doi:10.1136/bmjmilitary-2020-001482. [Epub ahead of print: 16 Apr 2020].

5 Loeffelholz MJ, Tang Y-W. Laboratory diagnosis of emerging human coronavirus infections - the state of the art. Emerg Microbes Infect 2020;9:747-56.

6 Buldú JM, Antequera DR, Aguirre J. The resumption of sports competitions after COVID-19 lockdown: the case of the Spanish football league. Chaos, Solitons \& Fractals 2020;138:109964.

7 Schemmerer M, Rauh C, Jilg W, et al. Time course of hepatitis E-specific antibodies in adults. J Viral Hepat 2017;24:75-9.

8 Gara N, Abdalla A, Rivera E, et al. Durability of antibody response against hepatitis B virus in healthcare workers vaccinated as adults. Clin Infect Dis 2015;60:505-13.

9 Stenger T, Ledo A, Ziller C, et al. Timing of vaccination after training: immune response and side effects in athletes. Med Sci Sports Exerc 2020;52:1603-9.

10 Schub D, Klemis V, Schneitler S, et al. High levels of SARS-CoV-2 specific T-cells with restricted functionality in patients with severe course of COVID-19, 2020. Available: https://medrxiv.org/cgi/content/short/2020.07.08.20148718v1 [Internet].
11 Robert Koch Institut. Robert Koch Institut [Internet]. Available: https://www.rki.de/DE/ Content/InfAZ/N/Neuartiges_Coronavirus/Fallzahlen.html

12 Knabl L, Tanmay M, Kimpel J, et al. High SARS-CoV-2 seroprevalence in children and adults in the Austrian ski resort Ischgl, 2020. Available: https://www.medrxiv.org/ content/10.1101/2020.08.20.20178533v1.full.pdf

13 Long Q-X, Tang X-J, Shi Q-L, et al. Clinical and immunological assessment of asymptomatic SARS-CoV-2 infections. Nat Med 2020;26:1200-4.

14 Knudsen NS, Thomasen MM, Andersen TB. Spread of virus during soccer matches, 2020. Available: https://www.medrxiv.org/content/10.1101/2020.04.26.20080614v1. full.pdf

15 Wölfel R, Corman VM, Guggemos W, et al. Virological assessment of hospitalized patients with COVID-2019. Nature 2020;581:465-9.

16 Yan Y, Chang L, Wang L. Laboratory testing of SARS-CoV, MERS-CoV, and SARSCoV-2 (2019-nCoV): current status, challenges, and countermeasures. Rev Med Virol 2020;30:e2106. 